\title{
Temporal generalization and peak shift in humans
}

\author{
LEWIS A. BIZO \\ Southern Cross University, Coffs Harbour, New South Wales, Australia \\ AND \\ Claire V. McMahon \\ University of Southampton, Southampton, England
}

\begin{abstract}
Three experiments investigated temporal generalization in humans. In Experiment 1, a peak shift effect was produced when participants were given intradimensional discrimination training. In Experiment 2, after training with a standard $\mathrm{S}+$ and generalization testing with an asymmetrical series of durations, generalization gradients moved toward the prevailing adaptation level. In Experiment 3, generalization gradients showed a central tendency shift and moved away from the $\mathrm{S}+$ and toward the mean of the test series (of 11 stimulus durations) after participants received training with $S+$ and $S-$ durations that in one condition were the 2 nd and 4 th and in another condition were the 4 th and 2 nd stimuli in the series, respectively. The results were inconsistent with an absolute account of peak shift but were consistent with an adaptation level account of peak shift.
\end{abstract}

A phenomenon central to our understanding of stimulus control is stimulus generalization. A classic demonstration of stimulus generalization was provided by Guttman and Kalish (1956), who reinforced pigeons' responding in the presence of a particular wavelength of light $(\mathrm{S}+)$ and then tested them in extinction with a range of stimuli that were evenly spaced around and included the $\mathrm{S}+$. The resulting generalization gradients showed that the pigeons had responded at the highest rate in the presence of the $\mathrm{S}+$ and at increasingly lower rates in the presence of other stimuli as the difference in wavelength from the $\mathrm{S}+$ increased. This procedure has formed the basis for most subsequent research on stimulus generalization. The degree to which the $\mathrm{S}+$ is discriminated from the $\mathrm{S}-$ is reflected in the steepness of the generalization gradient and is taken as a measure of stimulus control (for review, see Honig \& Urcuioli, 1981).

The peak of the generalization gradient is influenced by the methods employed to train the discrimination. Peak shift is a phenomenon associated with generalization when intradimensional discrimination training is given (see, e.g., Hanson, 1959). It is a general phenomenon that has been demonstrated with a number of species across a large number of stimulus dimensions (see reviews by Ghirlanda \& Enquist, 2003; Purtle, 1973). Typically, responding is reinforced in the presence of or after the presentation of one stimulus $(\mathrm{S}+)$ and is not reinforced in the presence of or after the presentation of some other stimulus $(\mathrm{S}-$ ). During postdiscrimination generalization testing, the peak of the generalization gradient is displaced from the $\mathrm{S}+$ to another stimulus that is farther from the $\mathrm{S}-$ than from the S+ (e.g., Hanson, 1959).

\section{Peak Shift Explained}

Two popular accounts of peak shift are Spence's (1937) explanation in terms of summation of excitatory and inhibitory gradients and Thomas's (e.g., Thomas, 1993; Thomas, Mood, Morrison, \& Wiertelak, 1991) explanation in terms of adaptation level theory (Helson, 1964).

Spence (1937) assumed that an excitatory gradient forms around the $\mathrm{S}+$, producing a tendency to respond in the presence of the $\mathrm{S}+$, and that this excitation of responding generalizes around the $\mathrm{S}+$. An inhibitory gradient is also assumed to form around the $\mathrm{S}-$, producing a tendency not to respond in the presence of the $\mathrm{S}-$, and this inhibition of responding is thought to generalize around the S- . Honig, Boneau, Burstein, and Pennypacker (1963) provided empirical evidence of both excitatory and inhibitory gradients following discrimination training. Spence's account assumes that observed generalization gradients result from the combination of inhibitory and excitatory tendencies to respond to the same stimulus. The summation of the inhibitory and excitatory gradients predicts the shift of a stimulus-generalization gradient from the $\mathrm{S}+$ in a direction away from the $\mathrm{S}$ - following intradimensional discrimination training.

Thomas (1993; Thomas et al., 1991) used Helson's (1964) adaptation level theory to explain peak shift, arguing that the relational aspects of stimuli determine the position of a generalization gradient. The stimuli used in

L.A. Bizo, lewis.bizo@scu.edu.au 
training an intradimensional discrimination are assumed to form adaptation levels or reference points for the discrimination, such that the $\mathrm{S}+$ is referenced as being $X$ units from this adaptation level (AL). The simplest assumption for the calculation of the AL is that it is the arithmetic mean of the stimuli that an individual has experienced. Thus, the AL is captured by Equation 1 (Thomas, 1993):

$$
\mathrm{AL}=Y(t)=\sum_{i=1}^{t} w(i) f[X(i)] .
$$

When novel stimuli are introduced during generalization testing, the $\mathrm{AL}$ changes to reflect the range of stimuli used, and invocation of the rule " $\mathrm{S}+$ is $X$ units from the $\mathrm{AL}$ " would result in responses to some novel stimulus that is now $X$ units from the new AL. Such a response strategy would produce peak shift.

Thomas (1993) outlined the basic assumptions of the model: The AL, $Y(t)$, is the average stimulus value. Stimuli are transformed from physical to subjective scales by a psychophysical function $f[X(i)]$. A weight parameter $w(i)$ determines the contribution of stimuli to the AL. Given these assumptions, it is possible to make predictions about the effects of different combinations of test stimuli that might be used in generalization testing on the AL.

It should be noted that Thomas (1993) also outlined an alternative form of the model based on the geometric mean, which makes the same ordinal predictions as does Equation 1. For the purposes of the present article, predictions of the AL account are based on the assumption that the prevailing $\mathrm{AL}$ is the arithmetic mean of the presented stimuli, and it is assumed that all stimuli attract equal weight. To illustrate the predictions of Equation 1, if one were to take a case where the training stimulus was the 9th stimulus from a set of 11 stimuli after some number of training trials $\left(n_{\text {tr }}\right)$, the AL would simply be the arithmetic mean of the stimulus number. For this example, the AL would be 9. If a participant then experienced some additional number of generalization trials $\left(n_{\mathrm{g}}\right)$, the calculated AL would simply be the arithmetic mean of all the stimuli that the participant experienced during training and generalization testing. Therefore, after 10 training trials and 22 generalization trials, the AL would be 6.94. The resulting generalization gradient that would be generated by this type of laboratory preparation would peak at a stimulus in the middle of a symmetrical set of test stimuli if the original $\mathrm{S}+$ was the middle stimulus of that set of test stimuli, and would peak at some stimulus other than the original $\mathrm{S}+$ if the test stimuli were asymmetrically distributed around the original $\mathrm{S}+$.

\section{Temporal Generalization}

A number of studies of stimulus generalization in both animals and humans have focused on stimulus duration (e.g., Church \& Gibbon, 1982; Church, Miller, Meck, \& Gibbon, 1991; Ferrara, Lejeune, \& Wearden, 1997; Wearden, 1991, 1992; Wearden, Denovan, Fakhri, \& Haworth, 1997; Wearden \& Towse, 1994). However, the motivation for these studies was not to test general models of generalization, but to test models specific to the temporal domain, as in, for example, Gibbon's (1977) scalar expectancy theory.
Consistent with research on other stimulus dimensions, when responding is reinforced in the presence of a particular stimulus duration $(\mathrm{S}+)$, generalization gradients peak at the S+. Church and Gibbon (1982) reinforced rats' leverpresses after the presentation of a particular stimulus duration ( $\mathrm{S}+$; a period of darkness) and not after shorter and longer stimulus durations, and the resulting generalization gradients peaked at the $\mathrm{S}+$ stimulus duration. This finding has been replicated with humans, using similar procedures for stimuli less than a second in duration and for stimuli in the range of several seconds. It is worth noting that these generalization gradients, however, tend to be skewed toward longer durations (see, e.g., Wearden, 1992; Wearden et al., 1997).

Demonstration of peak shift on a temporal dimension also has implications for the scalar property of timing. Psychometric functions from a variety of timing procedures tend to be superimposed when plotted as a function of relative time and coefficients of variation or Weber fraction are constant (see, e.g., Allan \& Gibbon, 1991; Church, 1993; Wearden, 1991). Peak shift would represent a violation of the scalar property of timing, since temporal generalization gradients would not be superimposed when plotted as a function of relative duration. Systematic variation from the superposition effect has been reported elsewhere, as when overall reinforcement density is manipulated (e.g., Bizo \& White, 1994a, 1994b, 1995, 1997). Hinton and Rao (2004) have also shown with humans that chronometric counting can disrupt the scalar property. Recently, Bizo, Chu, Sanabria, and Killeen (2006) have shown that Weber fractions were not constant and that they varied as a U-shaped function across a range of durations on separate temporal production and temporal categorization tasks.

In one study, Spetch and Cheng (1998) investigated peak shift with stimulus duration, yet this experiment did not produce peak shift. Pigeons' generalization gradients were best described as a step function following intradimensional discrimination training. The pigeons responded at low rates in the presence of the $\mathrm{S}-$ and all stimuli that were more similar to the $\mathrm{S}-$ than to the $\mathrm{S}+$, and they responded at high rates in the presence of the $\mathrm{S}+$ and all stimuli that were more similar to the $\mathrm{S}+$ than to the $\mathrm{S}-$. Spetch and Cheng (1998) suggested that the pigeons had responded as if the stimuli were categorical.

\section{EXPERIMENT 1}

In the present experiment, we attempted to produce a peak shift on a temporal generalization task. A go/no-go discrimination was used in a between-groups design. Participants were asked to respond only after presentation of the $\mathrm{S}+$ (the 6th stimulus from a geometric progression of 11 stimuli that differed by $20 \%$ apiece) and not to respond after any other stimulus presentation. In two other conditions, participants were given intradimensional discrimination training. They were asked to respond only after presentation of the $\mathrm{S}+$ (the 6th stimulus from the same progression of 11 stimuli) and were asked not to respond 
after presentation of the $\mathrm{S}-$ (either the 5 th or the 7 th stimulus from the series of 11 stimuli). The predictions of both accounts are summarized in Table 1. Both accounts predict that the peak of the gradient will shift from the $\mathrm{S}+$ in a direction away from the $\mathrm{S}-$. Spence's (1937) account predicts that following intradimensional discrimination training, the peak of the generalization gradient will shift to a stimulus value greater than the 6th stimulus for the $\mathrm{S} 6+\mathrm{S} 5-$ group and shift to a stimulus value less than the 6 th stimulus for the $\mathrm{S} 6+\mathrm{S} 7-$ group.

The relational account predicts that after the training phase, the AL would be 6 for the S6+ group, 5.5 for the $\mathrm{S} 6+\mathrm{S} 5-$ group, and 6.5 for the $\mathrm{S} 6+\mathrm{S} 7-$ group. The difference between the $\mathrm{S}+$ and the AL is $|0.5|$. Therefore, the response rule would be to respond to a stimulus that was equal to the AL plus 0.5 when the $\mathrm{S}-$ was the 5 th stimulus or respond to a stimulus that was equal to the AL minus 0.5 when the $\mathrm{S}-$ was the 7 th stimulus in the test series. During generalization testing, the AL would be the mean of all the stimulus values experienced during both training and testing. After the generalization testing phase, the AL is calculated to be 6.0 for the S6+ group, 5.9 for the S6+S5group, and 6.1 for the $\mathrm{S} 6+\mathrm{S} 7-$ group. The appropriate response during training was to respond to the $\mathrm{AL} \pm 0.5$. Applying the same rule during testing yields the predictions for the relational approach that are summarized in Table 1.

\section{Method}

Participants. The participants were 42 undergraduate students (mean age, 20.0 years; 12 were male and 30 female) at the University of Southampton who participated for course credit. Three more participants were excluded from the experiment because they made more than two errors during the training phase. Participants were randomly allocated to one of three conditions: S6+ $(n=14)$, $\mathrm{S} 6+\mathrm{S} 5-(n=14)$, and S6+S7- $(n=14)$.

Apparatus. Experimental events were controlled and recorded via a Pentium personal computer. Instructions were displayed on a 15-in. color monitor as black text on a white background. The stimuli consisted of timed presentations of a $9 \times 9 \mathrm{~mm}$ red square centered on the monitor. Stimulus durations were as follows: for $\mathrm{S} 1,0.38 \mathrm{sec} ; \mathrm{S} 2$, $0.46 \mathrm{sec} ; \mathrm{S} 3,0.55 \mathrm{sec} ; \mathrm{S} 4,0.66 \mathrm{sec} ; \mathrm{S} 5,0.79 \mathrm{sec} ; \mathrm{S} 6,0.95 \mathrm{sec} ; \mathrm{S} 7$, $1.14 \mathrm{sec} ; \mathrm{S} 8,1.37 \mathrm{sec} ; \mathrm{S} 9,1.65 \mathrm{sec} ; \mathrm{S} 10,1.98 \mathrm{sec}$; and S11, $2.37 \mathrm{sec}$.

Participants' responses were recorded via a response box with two buttons, one labeled "Yes" and the other "No," that was connected to the computer via the serial port. Participants were required to respond by pressing "Yes" after each presentation of the $\mathrm{S}+$. The "No" button was not used in this experiment.

Procedure. At the start of the experiment, the participants were informed that they were participating in an experiment on time perception and that they would have to differentiate standard "test" durations from other durations. Participants were then shown the "test" duration. In each of the three conditions, participants were

Table 1

Predicted Peak of Generalization Gradients for the Absolute and Relational Accounts of Peak Shift for Experiment 1

\begin{tabular}{lccc}
\hline & \multicolumn{3}{c}{ Group } \\
\cline { 2 - 4 } Account & $\mathrm{S} 6+\mathrm{S} 5-$ & $\mathrm{S} 61+$ & $\mathrm{S} 6+\mathrm{S} 7-$ \\
\hline Absolute & $>6$ & 6 & $<6$ \\
Relational & $6.4(5.9+0.5)$ & $6(6)$ & $5.6(6.1-0.5)$ \\
\hline
\end{tabular}

Note-The predicted adaptation levels from the relational account are given in parentheses. instructed to press "Yes" following each presentation of the test duration, which was $0.95 \mathrm{sec}(\mathrm{S} 6+)$. Two groups received intradimensional discrimination training that lasted for 24 trials. The $\mathrm{S}+$ and $\mathrm{S}$ - were each presented 12 times, in a random order. One of these groups was instructed to press "Yes" after stimulus presentations of $0.95 \mathrm{sec}(\mathrm{S} 6+)$ and not to respond after stimulus presentations of $0.79 \mathrm{sec}$ (S5-). Another group was instructed to press "Yes" after stimulus presentations of $0.95 \mathrm{sec}(\mathrm{S} 6+)$ and not to respond after stimulus presentations of $1.14 \mathrm{sec}(\mathrm{S} 7-)$. A third group did not receive intradimensional discrimination training; for this group, the training phase consisted of 12 presentations of the $\mathrm{S}+$, the same number of presentations of the $\mathrm{S}+$ as that which the other two groups experienced. The word "Correct" or "Incorrect" was presented to participants on the monitor following correct or incorrect presses of the "Yes" button during the training phase. If participants made more than two errors in the last 8 training trials, they received an additional 8 training trials. If they made more than two errors in these additional training trials, their participation in the experiment was terminated. The group that did not receive intradimensional discrimination training saw the word "Correct" only as feedback for pressing "Yes." The words "Training Phase" were positioned at the top of the screen throughout the training phase.

After the training trials, the test phase began. The test phase was exactly the same for all three groups. The words "Test Phase" were positioned at the top of the screen throughout the test phase. The sequence of 66 test phase trials was different for each participant, with the complete series of 11 stimuli $(\mathrm{S} 1-\mathrm{S} 11)$ presented in six randomized blocks. Participants had earlier been instructed to continue pressing the "Yes" button on the response box following each presentation of the test duration during the test phases and were told that responses would no longer be followed by feedback. The experimenter was in the room throughout the experiment, seated behind and out of view of participants. Each participant experienced two sessions. Sessions were separated by a short break. The probability of reporting a stimulus to be of the test duration was calculated by dividing the number of "test duration" responses by the number of opportunities to make such a response.

\section{Results}

The mean generalization gradients for each of the conditions are shown in Figure 1. When participants were asked to respond following the presentation of the $\mathrm{S}+$, the resulting generalization gradient peaked at the $\mathrm{S}+$. When participants were provided with intradimensional discrimination training, the peak probability of reporting any duration as the $\mathrm{S}+$ duration shifted to a stimulus other than the original training stimulus in a direction away from the $\mathrm{S}+$ and even further from the $\mathrm{S}-$.

The mean of each generalization gradient was calculated for each individual for each condition. The means and standard deviations for each group's generalization gradients were as follows: $\mathrm{S} 6+, M=6.30, S D=0.95$; $\mathrm{S} 6+\mathrm{S} 5-, M=7.01, \mathrm{~S} D=0.89 ; \mathrm{S} 6+\mathrm{S} 7-, M=5.65$, $S D=1.01$. A one-way ANOVA confirmed that there was a significant difference in the means across the three groups $[F(2,39)=6.97, p<.05]$. Planned comparison independent $t$ tests confirmed the directions of the peak shift effects shown in Figure 1. There was a significant difference between the means for the S6+ group and the means for the $\mathrm{S} 6+\mathrm{S} 5-$ group $[t(26)=2.01, p<.05]$; a significant difference between the means for the $\mathrm{S} 6+$ group and the means of the $\mathrm{S} 6+\mathrm{S} 7-$ group $[t(26)=1.74$, $p<.05]$; and a significant difference between the means for the S6+S5 - group and the means for the S6+S7- 


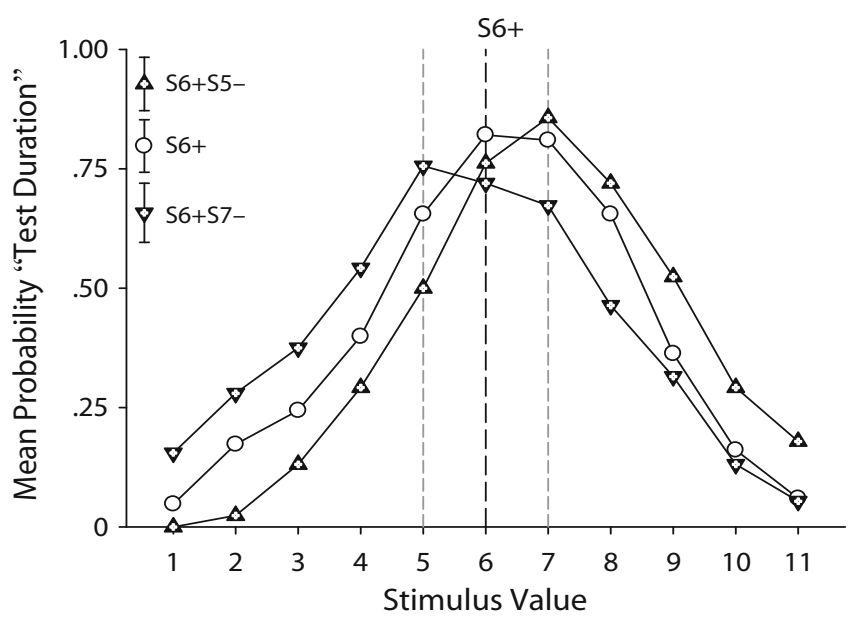

Figure 1. The mean probability of reporting a stimulus as being of the test duration is plotted against stimulus value. Generalization gradients were produced by averaging across individual participants for each of the three groups (S6+, S6+S5-, S6+S7-). The average standard errors of the mean are shown in the figure legend.

group $[t(26)=3.73, p<.05]$. The means predicted by the adaptation level account did not differ significantly from the obtained means for the $\mathrm{S} 6+[t(13)=1.20, p>.05]$ or $\mathrm{S} 6+\mathrm{S} 7-[t(13)=0.10, p>.05]$ groups, but did differ significantly from the obtained means for the $\mathrm{S} 6+\mathrm{S} 5-$ group $[t(13)=2.66, p<.05]$. It should be noted for this group that the observed peak shift was larger than predicted by the adaptation level account (7.01 vs. 6.4). However, if the weight parameter were free to take values other than 1 , that would bring the predicted means into line with those observed.

In the present experiment, during the training phase, only responses following presentation of the $\mathrm{S}+$ were followed by feedback. When responses were withheld following presentations of the $\mathrm{S}-$, they were not followed by any feedback. This may have increased the overall level of responding or a bias to respond "Yes"; however, it would be reasonable to expect this tendency to be distributed evenly around the $\mathrm{S}+$.

\section{EXPERIMENT 2}

In Experiment 2, the effect of a skewed stimulus range on temporal generalization was investigated. On other stimulus dimensions, asymmetrical series of test stimuli have been shown to produce a central tendency shift. Thomas and Jones (1962), with wavelengths of light, and Helson and Avant (1967), with stimulus size, have shown that following exposure to a skewed distribution of test stimuli, participants respond more to stimuli closer to the $\mathrm{AL}$ or to the mean of the test series than they respond to the original $\mathrm{S}+$. In the present experiment, we attempted to produce a central tendency shift with stimulus duration. The same general procedure as used in Experiment 1 was used in Experiment 2: A go/no-go discrimination was used. Participants were asked to respond only after presentation of the S+, which was the same for both conditions, and not to respond after any other stimulus presentation. Fifteen test stimuli were used in total, but only 11 test stimuli were used in each condition. In one condition, the $\mathrm{S}+$ was the 8th stimulus in a series of 11 stimuli, and in the other condition, the $\mathrm{S}+$ was the 4th stimulus in another series of 11 stimuli. The asymmetrical positioning of the $\mathrm{S}+$ in the series of 11 stimuli used during generalization testing was expected to produce a central tendency effect, such that the peak of the gradient should shift from the $\mathrm{S}+$ in a direction toward the prevailing AL. The predicted AL after presentation of any given sequence of stimuli would be the mean of those stimuli weighted by the number of presentations of each stimulus in the set of stimuli. When the $\mathrm{S}+$ was either the 4 th or the 8 th stimulus, the AL after the 12 training trials was 8.0 for each condition. After the 66 generalization test trials, the AL was 9.7 and 6.3. The resulting generalization gradients for each condition should move toward the AL and away from the S+. Spence's absolute account did not predict this shift.

\section{Method}

Participants. The participants were 18 undergraduate students at the University of Southampton (mean age, 20.0 years; 12 were male and 6 female) who participated for course credit.

Apparatus and Procedure. The apparatus was the same as that used in Experiment 1. The general procedure was similar to that used for the group in Experiment 1 that experienced presentations of the $\mathrm{S}+$ only during training and did not receive intradimensional discrimination training. In Experiment 2, participants experienced two separate conditions in a within-groups design, and the order of exposure was counterbalanced across participants. Participants were asked to respond only after presentation of the $\mathrm{S}+$, which was always $0.72 \mathrm{sec}$, and not to respond after any other stimulus presentation. Participants were given 12 presentations of the $\mathrm{S}+$ before they were exposed to generalization testing. This was sufficient exposure to the $\mathrm{S}+$ to produce a relatively symmetrical generalization gradient in Experiment 1 for the group that did not receive intradimensional discrimination training. During generalization testing for one condition, the $\mathrm{S}+$ was the 8 th stimulus in a geometric progression of 11 stimuli that differed by $25 \%$ and that ranged from 0.15 to $1.40 \mathrm{sec}$. For the other condition, the $\mathrm{S}+$ was the 4 th stimulus in a geometric progression of 11 stimuli that differed by $25 \%$ and that ranged from 0.37 to $3.41 \mathrm{sec}$. The 15 test stimuli were distributed around the $\mathrm{S}+$. The stimulus durations were as follows: for $\mathrm{S} 1,0.15 \mathrm{sec} ; \mathrm{S} 2,0.19 \mathrm{sec} ; \mathrm{S} 3$, $0.23 \mathrm{sec} ; \mathrm{S} 4,0.29 \mathrm{sec} ; \mathrm{S} 5,0.37 \mathrm{sec} ; \mathrm{S} 6,0.46 \mathrm{sec} ; \mathrm{S} 7,0.57 \mathrm{sec} ; \mathrm{S} 8$, $0.72 \mathrm{sec}$ (the $\mathrm{S}+$ ); S9, $0.89 \mathrm{sec} ; \mathrm{S} 10,1.12 \mathrm{sec} ; \mathrm{S} 11,1.40 \mathrm{sec} ; \mathrm{S} 12$, $1.75 \mathrm{sec} ; \mathrm{S} 13,2.18 \mathrm{sec} ; \mathrm{S} 14,2.73 \mathrm{sec}$; and S15, $3.41 \mathrm{sec}$. There were 66 generalization trials in each condition. Stimulus durations were presented six times each in each of six blocks of randomized trials.

\section{Results}

The averaged generalization gradients for each condition are shown in Figure 2. To facilitate comparison of the two conditions, the means of the generalization gradient were calculated using the ordinal positions of the $\mathrm{S}+$ and test stimuli from the full set of 15 stimuli. The mean of each generalization gradient was calculated for each individual in each condition. Although the $\mathrm{S}+$ had the same absolute duration in both conditions, the means for each condition were significantly different: $\mathrm{S} 4+, M=8.64$, $S D=0.94$, and $\mathrm{S} 8+, M=7.46, S D=0.61[t(18)=$ $5.05, \mathrm{p}<.05]$. The means had moved in a direction away 
from the $\mathrm{S}+$ (the 8th stimulus in the full set of 15 test stimuli) and toward the prevailing AL, thus demonstrating a central tendency effect.

The means of the generalization gradients predicted by the adaptation level account were compared against the obtained means. The predicted means were significantly larger than the obtained means for the S4+ condition $[t(18)=4.91, p<.05]$ and significantly smaller than the obtained means for the $\mathrm{S} 8+$ condition $[t(18)=8.28, p<$ $.05]$. The magnitude of the central tendency shift was not as great as that predicted by the AL account.

\section{EXPERIMENT 3}

In Experiment 1, both the absolute and relational accounts predicted the direction of the peak shift effects, and the predictions of the adaptation level account were remarkably close to the observed shifts for two of three conditions. In Experiment 2, asymmetrical test series skewed the resulting generalization gradients toward the prevailing AL in agreement with predictions of adaptation level theory.

Other experiments have shown that under certain conditions, relative and absolute accounts of peak shift make conflicting predictions. Thomas, Windell, Williams, and White (1985) showed that the peak of the generalization gradient moved toward the $\mathrm{S}-$ and away from the $\mathrm{S}+$ when they varied the frequency of presentation of the $\mathrm{S}+$ and $\mathrm{S}-$ relative to other stimuli used during generalization testing with stimuli that differed along the dimension of brightness. The gradient moved toward the stimulus that had been presented the most often during generalization testing, as was consistent with an AL account, but inconsistent with an absolute account, which would predict that the gradient should move away from and not toward the $\mathrm{S}-$.

As Thomas (1993) has pointed out, the most compelling experiment that would differentiate between absolute and relational accounts is one where the AL would be closer to the $\mathrm{S}-$ than to the $\mathrm{S}+$, which would lead to the counterintuitive prediction that participants would respond to stimuli that were more similar to the $\mathrm{S}-$ than to the $\mathrm{S}+$. Such a preparation was reported by Thomas et al. (1991), who detailed the results of an experiment where Spence's (1937) model and the adaptation level model made opposite predictions (Figure 3). Thomas et al. (1991) required one group of participants to respond to the 2 nd stimulus of a set of 11 stimuli (different light intensities) and not to respond to the 4th stimulus from the same set of stimuli, and required another group of participants to respond to the 4 th stimulus of a set of 11 stimuli and not to respond to the 2 nd stimulus from the same set of stimuli.

Spence's (1937) account would predict that the S2+S4group that was trained to respond to the 2 nd stimulus but not the 4th would produce a generalization gradient that was shifted to a stimulus value less than that of the 2 nd stimulus and that the $\mathrm{S} 2-\mathrm{S} 4+$ group trained to respond to the 4 th stimulus but not the 2 nd would produce a generalization gradient shifted to a stimulus value greater than that of the 4th stimulus.

The adaptation level account makes predictions that stand in contrast to those of Spence. After the train-

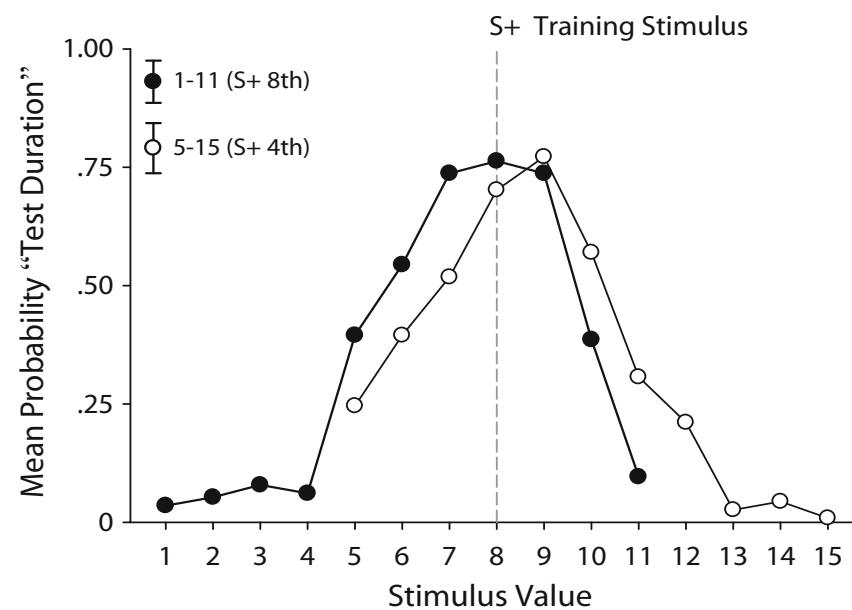

Figure 2. Generalization gradients for two conditions in which participants were given training with the same $\mathrm{S}+$ (training stimulus), but were tested with two different series of stimuli during generalization testing. The stimuli used during testing were skewed to be either predominantly shorter or longer than the $\mathrm{S}+$. The average standard errors of the mean are shown in the figure legend.

ing phase, the $\mathrm{AL}$ was 3 for the $\mathrm{S} 2+\mathrm{S} 4-$ and $\mathrm{S} 2-\mathrm{S} 4+$ groups. The appropriate response would be to respond to the AL minus 1 for the $\mathrm{S} 2+\mathrm{S} 4-$ group and respond to the AL plus 1 for the $\mathrm{S} 2-\mathrm{S} 4+$ group. The AL was assumed to shift toward the mean of the total set of stimuli shown during training and testing. During testing, a range of stimuli were presented; these were skewed to intensities greater than those of the original $\mathrm{S}+$ and $\mathrm{S}-$. Consequently, after the training phase the AL was 5.2 for the $\mathrm{S} 2+\mathrm{S} 4-$ and $\mathrm{S} 2-\mathrm{S} 4+$ groups. Thus, when the response

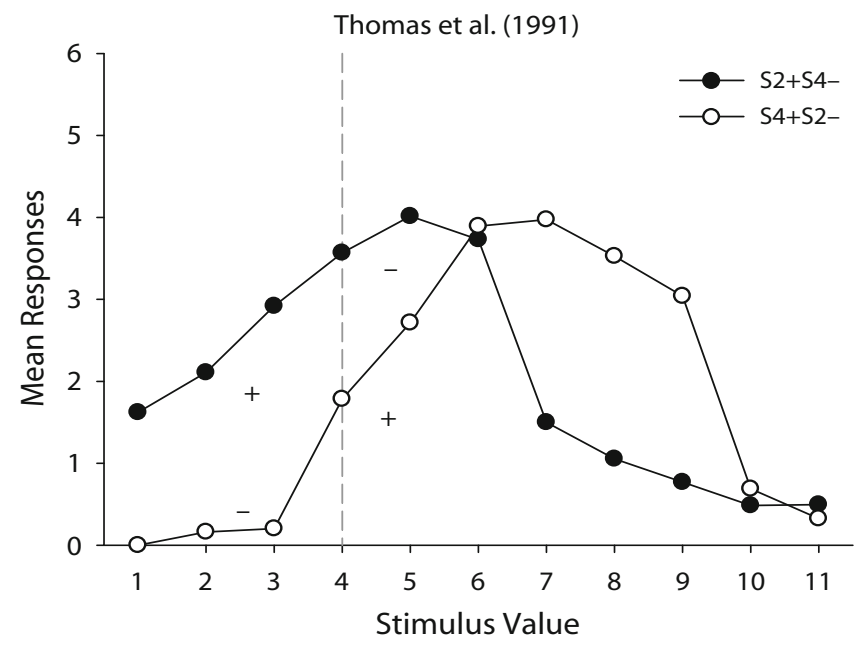

Figure 3. Mean responses plotted as a function of stimulus value. The "+" and " -" signs indicate whether the second or fourth stimulus was the $\mathrm{S}+$ or $\mathrm{S}-$. From "Peak Shift Revisited: A Test of Alternative Interpretations," by D. R. Thomas, K. Mood, S. Morrison, \& E. Wiertelak, 1991, Journal of Experimental Psychology: Animal Behavior Processes, 17, p. 133. Copyright 1991 by the American Psychological Association. Adapted with permission. 
rule is applied, the adaptation level model would predict that the peak of the generalization gradients should shift to 4.2 for the $\mathrm{S} 2+\mathrm{S} 4-$ group and to 6.2 for the $\mathrm{S} 2-\mathrm{S} 4+$ group (see Table 2). For the $\mathrm{S} 2+\mathrm{S} 4-$ group, the peak should move toward and not away from the $\mathrm{S}-$.

The data from Thomas et al. (1991) are reproduced in Figure 3. Thomas et al. (1991) found that the generalization gradients shifted in a direction consistent with an adaptation level account; the peaks of the gradients were larger than 2 for both groups, and this is inconsistent with predictions of Spence's absolute account.

The design of Experiment 3 was based on that used by Thomas et al. (1991). If temporal generalization is skewed by the presentation of an asymmetrical series of stimuli during generalization testing, then the resulting generalization gradients should show a central tendency shift or range effect as illustrated by Thomas et al. (1991; see Figure 3). If judgments about stimulus duration are based on absolute stimulus properties, however, then the resulting generalization gradients should show evidence of peak shift: The peak of the gradient should always be shifted from the $\mathrm{S}+$ in a direction away from the $\mathrm{S}-$.

\section{Method}

Participants. The participants were 20 undergraduate and postgraduate students at the University of Southampton (mean age, 25.7 years; 13 were male and 7 female) who were paid $5 £$ sterling for their participation.

Apparatus and Procedure. The experimental apparatus was the same as that used in Experiments 1 and 2. The procedure was similar to that used in Experiment 1, except that in one condition, participants were instructed to press the "Yes" button after stimulus presentations of $0.46 \mathrm{sec}(\mathrm{S} 2+)$ and were instructed not to respond after stimulus presentations of $0.66 \mathrm{sec}(\mathrm{S} 4-)$. In a second condition, participants were instructed to press the "Yes" button after stimulus presentations of $0.66 \mathrm{sec}(\mathrm{S} 4+)$ and were instructed not to respond after stimulus presentations of $0.46 \mathrm{sec}$ (S2-). Each participant experienced two experimental sessions, and the order of exposure to the two conditions was counterbalanced across participants. Experimental sessions were conducted on consecutive days at approximately the same time of day.

\section{Results and Discussion}

The generalization data for individual participants were averaged separately for the two conditions and are presented in Figure 4. Participants who were trained to respond to the $\mathrm{S} 2+$ and not to respond to the $\mathrm{S} 4-$ subsequently responded during generalization testing nearly as often to the $\mathrm{S}-$ as they did to the $\mathrm{S}+$. This effect is not predicted by Spence's (1937) account of peak shift, but it is consistent with the predictions of an adaptation

Table 2

Predicted Peak of Generalization Gradients for the Absolute and Relational Accounts of Peak Shift for Experiment 3

\begin{tabular}{ccc}
\hline & \multicolumn{2}{c}{ Condition } \\
\cline { 2 - 3 } Account & $\mathrm{S} 2+\mathrm{S} 4-$ & $\mathrm{S} 2-\mathrm{S} 4+$ \\
\hline Absolute & $<2$ & $>4$ \\
Relational & $4.2(5.2)$ & $6.2(5.2)$ \\
\hline
\end{tabular}

Note-The predicted adaptation levels from the relational account are given in parentheses. level account of peak shift. The pattern of results shown in Figure 4 is comparable to the pattern of results reported by Thomas et al. (1991) shown in Figure 3.

Predictions of the relational account and the AL account were compared against the obtained means. The critical condition in this experiment was the $\mathrm{S} 2+\mathrm{S} 4-$ condition, where the two accounts predicted that the peaks of the gradients would move in opposite directions. The means were greater than 2 for 19 of the 20 participants in the $\mathrm{S} 2+\mathrm{S} 4-$ condition; this is consistent with the AL account but not Spence's account. The means were greater than 4 for 18 of 20 participants in the $\mathrm{S} 2-\mathrm{S} 4+$ condition. The peaks of the gradients shifted toward the prevailing AL in both conditions. The magnitude of the central tendency shift, however, was not as great as that predicted by the AL account in both conditions. The predicted means were significantly larger than the obtained means for the $\mathrm{S} 2+\mathrm{S} 4-$ condition $[t(19)=4.75, p<.05]$ and were not significantly smaller than the obtained means for the $\mathrm{S} 2+\mathrm{S} 4-$ condition $[t(19)=1.43, p>.05]$.

\section{GENERAL DISCUSSION}

Peak shift was observed in Experiment 1. The peaks of temporal generalization gradients proved to be susceptible to the influence of skewed stimulus ranges in Experiments 2 and 3. In Experiment 3, the adaptation level approach predicted more responses to the third stimulus (S3) than to the first stimulus (S1) when participants experienced the second stimulus as the $\mathrm{S}+$ and the fourth stimulus as the $\mathrm{S}-$. The peaks of generalization gradients moved toward the mean of the stimulus set used during generalization testing following intradimensional discrimination training. The direction of the shift of the gradients was consistent with an adaptation level account of peak shift, such as that offered by Thomas. The results of Experiment 3 in particular show the strongest support for a relational account of peak shift. The two accounts make opposite predictions, and the data from the present experiment clearly support the predictions of the AL approach. The counterintuitive prediction that under certain conditions participants would be more inclined to respond to a stimulus more like the $\mathrm{S}-$ than like the S+ was confirmed, and this replicated the findings of Thomas et al. (1991) for light intensity.

The observed central tendency shifts in both conditions of Experiment 2 and in one condition of Experiment 3, though in the predicted direction, were significantly smaller in magnitude than AL theory predicted. Elsewhere, Thomas has argued that adaptation should develop gradually during testing (e.g., Thomas, 1993; Thomas et al., 1991). The mismatch of data with predictions usually occurs because of the assumptions underlying their generation. It may be that participants give more weight to training stimuli that are associated with feedback than they do to stimuli that are not. This could be tested by varying relative numbers of training and test stimuli while maintaining a constant AL.

It has been observed elsewhere in humans that temporal generalization gradients can be asymmetrical, being skewed toward longer durations, particularly when lin- 


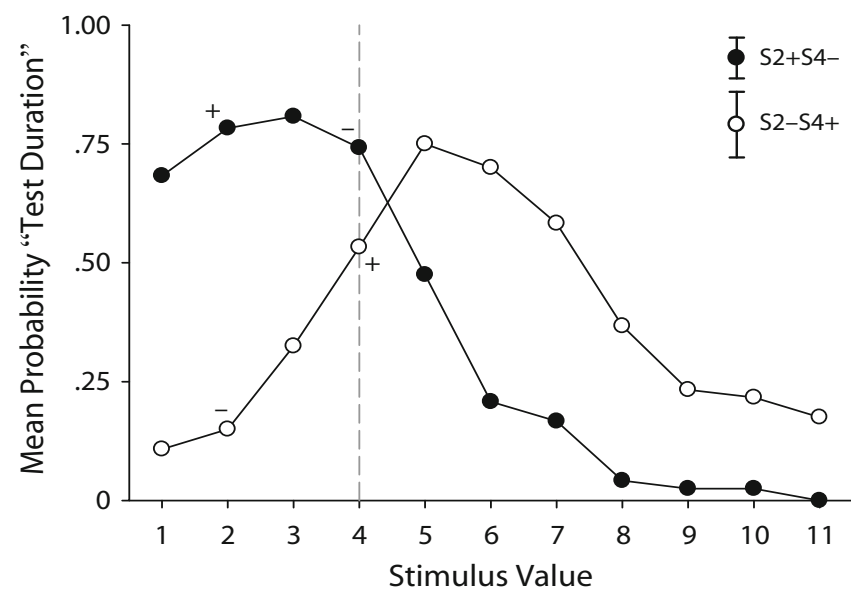

Figure 4. The mean probability of reporting a stimulus as being of the test duration, plotted against stimulus value. Generalization gradients were produced by averaging individual participants for each condition ( $\mathrm{S} 2-\mathrm{S} 4+$ and $\mathrm{S} 2+\mathrm{S} 4-)$. The average standard errors of the mean are shown in the figure legend.

ear rather than logarithmic progressions are used (e.g., Wearden, 1992; Wearden et al., 1997). The implication for the present experiments is that it may have been easier to demonstrate a peak shift toward longer rather than shorter durations. Indeed in the present Experiment 1, the mean of the generalization gradient for the $\mathrm{S}+$ only condition was 0.3 larger than that for the $\mathrm{S}+$ condition, and the difference between the two $\mathrm{S}+\mathrm{S}-$ conditions showed a greater shift for the condition that pushed the peak to longer stimulus durations. In Experiment 2, the effect of stimulus range was greater when the $\mathrm{S}+$ was at the short rather than the long end of stimulus durations, which was again consistent with the general asymmetry of temporal generalization gradients. Despite the presence of this potential confound, however, a peak shift was still observed toward shorter durations.

The effect of stimulus range on generalization performance is important for other reasons. There are numerous examples of peak shift in humans that can be attributed to the range and manner of presentation of stimuli during generalization testing (for a review, see Thomas, 1993). The assumption of a change in adaptation level is sufficient to account for the majority of these data. Peak shift, however, cannot always be attributed to changes in adaptation level. Recently, peak shift has been demonstrated in humans by Cheng and Spetch (2002) with spatial stimuli when adaptation level was kept constant. Cheng and Spetch required participants to bet on stimuli that were positioned at different spatial locations and were able to produce peak and area shifts in a series of experiments. Cheng and Spetch argued that peak shift has been tested primarily with stimuli that vary along a single dimension and that these discriminations are susceptible to range effects (e.g., Thomas, 1993); complex stimuli such as spatial location or faces, however, are not (e.g., Spetch, Cheng, \& Clifford, 2004).

The only other attempt to demonstrate peak shift with duration of stimuli failed to produce a peak shift. Spetch and Cheng (1998), with pigeons, found that the generalization gradients that the birds produced resembled step functions best. The birds responded at low rates in the presence of the $\mathrm{S}-$ and all stimuli that were more similar to the $\mathrm{S}-$ than to the $\mathrm{S}+$ and responded at high rates in the presence of the $\mathrm{S}+$ and all stimuli that were more similar to the $\mathrm{S}+$ than to the $\mathrm{S}-$. They suggested that the pigeons had formed categories and had treated all stimuli as if they were either the $\mathrm{S}+$ or the $\mathrm{S}-$. Why, then, the difference between their results and ours? In their discussion, Spetch and Cheng offered a number of plausible factors that might have produced the step functions observed in their experiments, such as expectancies formed during training and restriction of training to just two stimulus durations. There are numerous methodological differences between our experiments and theirs, and to point to a species difference would be trivial. The most important factor is probably that we could give our human participants explicit instructions, and although they did receive a short period of training, they could discriminate between the $\mathrm{S}+$ and $\mathrm{S}-$ after just 24 trials, whereas the pigeons in Spetch and Cheng's study experienced 2,640 trials (48 trials per session $\times 55$ sessions)

Elsewhere, White and Thomas (1979b) have shown that when pigeons could respond during the $\mathrm{S}+$ presentation, the resulting generalization gradients did not show a peak shift, but did show a peak shift when the pigeons could not make those responses, although the gradients were still peaked sharply at or near the $\mathrm{S}+$. White and Thomas (1979a) have shown in humans that increasing the range of stimulus values used during generalization testing can generate a peak shift effect whereas a reduced stimulus range does not. They argued that increasing the range of test stimuli might help to overcome the use of relational strategies that categorize test stimuli.

Our generalization tests did not provide any feedback to participants, whereas Spetch and Cheng (1998) interspersed reinforced $\mathrm{S}+$ trials among the test trials, although in one experiment generalization testing was conducted in extinction. Perhaps the continued presence of reinforcement and the extended training and testing encouraged the pigeons to treat the durations as belonging to one of two categories, either "S+"-like or "S-"-like. If the pigeons had formed categories, perhaps by using interim behaviors to mediate their timing performance, as has been suggested and has been shown elsewhere to mediate accuracy on temporal discriminations (e.g., Harper \& Bizo, 2000; Killeen \& Fetterman, 1988; Killeen, Fetterman, \& Bizo, 1997), then one would expect a pattern of results similar to that found by Spetch and Cheng.

Although the present experiments were not designed or conceived to test between internal clock models and theories of stimulus generalization, they do present a challenge to the scalar property of timing. Peak shift and central tendency effects on a temporal dimension when the $\mathrm{S}+$ is held constant violate the scalar property of timing. Bizo et al. (2006) recently reported data showing systematic deviation from Weber's law, which they accounted for by assuming countererror (see Killeen \& Taylor, 2000). It is possible that the asymmetrical test series may be differentially af- 
fected by countererror, and this might in part explain why temporal generalization gradients are frequently skewed to longer durations, with participants showing higher probabilities of responding to stimuli longer than the $\mathrm{S}+$ than to stimuli shorter than the S+ (e.g., Wearden et al. 1997).

In conclusion, the present experiments are the first to show a central tendency effect on a temporal dimension. These experiments further demonstrate the general nature of this phenomenon. As mentioned, earlier peak shift has been observed across a wide variety of stimulus dimensions and across a variety of species. AL theory predicted the direction of the shifts for all experiments, whereas Spence's absolute account did not. However, the AL account tended to overestimate the predicted shift. This might have been caused by participants mediating their timing by counting, which has been shown elsewhere to improve timing accuracy. If participants formed discrete temporal categories, this would attenuate the central tendency effect, and this warrants further investigation.

\section{AUTHOR NOTE}

Experiment 1 was reported in partial fulfillment of the requirements for the BSc (Hons) degree by C.V.M. Address correspondence to L. A. Bizo, Department of Psychology, Southern Cross University, Coffs Harbour, NSW 2450, Australia (e-mail: lewis.bizo@scu.edu.au).

\section{REFERENCES}

Allan, L. G., \& Gibbon, J. (1991). Human bisection at the geometric mean. Learning \& Motivation, 22, 39-58.

Bizo, L. A., Chu, J. Y. M., Sanabria, F., \& Killeen, P. R. (2006). The failure of Weber's law in time perception and production. Behavioural Processes, 71, 201-210.

Bizo, L. A., \& White, K. G. (1994a). Behavioral theory of timing: Reinforcement rate determines pacemaker rate. Journal of the Experimental Analysis of Behavior, 61, 19-33.

Bizo, L. A., \& White, K. G. (1994b). Pacemaker rate in the behavioral theory of timing. Journal of Experimental Psychology: Animal Behavior Processes, 20, 308-321.

Bizo, L. A., \& White, K. G. (1995). Reinforcement context and pacemaker rate in the behavioral theory of timing. Animal Learning \& Behavior, 23, 376-382.

Bizo, L. A., \& White, K. G. (1997). Timing with controlled reinforcer density: Implications for models of timing. Journal of Experimental Psychology: Animal Behavior Processes, 23, 44-55.

Cheng, K., \& Spetch, M. L. (2002). Spatial generalization and peak shift in humans. Learning \& Motivation, 33, 358-389.

Church, R. M. (1993). Human models of animal behavior. Psychological Science, 4, 170-173.

Church, R. M., \& Gibbon, J. (1982). Temporal generalization. Journal of Experimental Psychology: Animal Behavior Processes, 8 , 165-186.

Church, R. M., Miller, K. D., Meck, W. H., \& Gibbon, J. (1991). Symmetrical and asymmetrical sources of variance in temporal generalization. Animal Learning \& Behavior, 19, 207-214.

Ferrara, A., Lejeune, H., \& Wearden, J. H. (1997). Changing sensitivity to duration in human scalar timing: An experiment, a review, and some possible explanations. Quarterly Journal of Experimental Psychology, 50B, 217-237.

Ghirlanda, S., \& ENQuist, M. (2003). A century of generalization. Animal Behaviour, 66, 15-36.

GibBon, J. (1977). Scalar expectancy theory and Weber's law in animal timing. Psychological Review, 84, 279-325.

Guttman, N., \& Kalish, H. I. (1956). Discriminability and stimulus generalization. Journal of Experimental Psychology, 51, 79-88.
HANSON, H. M. (1959). Effects of discrimination training on stimulus generalization. Journal of Experimental Psychology, 58, 321-334.

HARPER, D. N., \& Bizo, L. A. (2000). Mediation of timing accuracy by operant behavior. Behavioural Processes, 50, 143-154.

HeLson, H. (1964). Adaptation level theory. New York: Harper \& Row.

Helson, H., \& Avant, L. L. (1967). Stimulus generalization as a function of contextual stimuli. Journal of Experimental Psychology, 73, 565-567.

Hinton, S. C., \& Rao, S. M. (2004). "One-thousand one ... onethousand two ...": Chronometric counting violates the scalar property in interval timing. Psychonomic Bulletin \& Review, 11, 24-30.

Honig, W. K., Boneau, C. A., Burstein, K. R., \& Pennypacker, H. S. (1963). Positive and negative generalization gradients obtained under equivalent training conditions. Journal of Comparative \& Physiological Psychology, 56, 111-116.

Honig, W. K., \& Urcuioli, P. J. (1981). The legacy of Guttman and Kalish (1956): 25 years of research on stimulus generalization. Journal of the Experimental Analysis of Behavior, 36, 405-445.

KilleEn, P. R., \& Fetterman, J. G. (1988). A behavioral theory of timing. Psychological Review, 95, 274-295.

Killeen, P. R., Fetterman, J. G., \& Bizo, L. A. (1997). Time's causes. In C. M. Bradshaw \& E. Szabadi (Eds.), Time and behaviour: Psychological and neurobehavioural analyses (Advances in Psychology, Vol. 120, pp. 79-131). Amsterdam: Elsevier.

KilleEN, P. R., \& TAYLOR, T. J. (2000). How the propagation of error through stochastic counters affects time discrimination and other psychophysical judgments. Psychological Review, 107, 430-459.

Purtle, R. B. (1973). Peak shift: A review. Psychological Bulletin, 80, 408-421.

SPEnCE, K. W. (1937). The differential response in animals to stimuli varying within a single dimension. Psychological Review, 44, 430-444.

SPetch, M. L., \& Cheng, K. (1998). A step function in pigeons' temporal generalization in the peak shift task. Animal Learning \& Behavior, 26, $103-118$

Spetch, M. L., Cheng, K., \& Clifford, C. W. G. (2004). Peak shift but not range effects in recognition of faces. Learning \& Motivation, 35, 221-241.

Thomas, D. R. (1993). A model of adaptation-level effects on stimulus generalization. Psychological Review, 100, 658-673.

Thomas, D. R., \& Jones, C. G. (1962). Stimulus generalization as a function of the frame of reference. Journal of Experimental Psychology, 64, 77-80.

Thomas, D. R., Mood, K., Morrison, S., \& Wiertelak, E. (1991). Peak shift revisited: A test of alternative interpretations. Journal of Experimental Psychology: Animal Behavior Processes, 17, 130-140.

Thomas, D. R., Windell, B. T., Williams, J. L., \& White, K. G. (1985). Stimulus presentation frequency in brightness discrimination and generalization: A test of adaptation-level and signal-detection interpretations. Perception \& Psychophysics, 37, 243-248.

WEARDEN, J. H. (1991). Do humans possess an internal clock with scalar timing properties? Learning \& Motivation, 22, 59-83.

WEARDEN, J. H. (1992). Temporal generalization in humans. Journal of Experimental Psychology: Animal Behavior Processes, 18, 134-144.

Wearden, J. H., Denovan, L., FaKhri, M., \& Haworth, R. (1997) Scalar timing in temporal generalization in humans with longer stimulus durations. Journal of Experimental Psychology: Animal Behavior Processes, 23, 502-511.

Wearden, J. H., \& Towse, J. H. (1994). Temporal generalization in humans: Three further studies. Behavioural Processes, 32, 247-264.

White, K. G., \& Thomas, D. R. (1979a). Postdiscrimination stimulus generalization in humans: An extension of Galizio and Baron. Animal Learning \& Behavior, 7, 564-565.

White, K. G., \& Thomas, D. R. (1979b). Topographically tagged stimulus control: Maintained generalization and stimulus-specific gradients. Bulletin of the Psychonomic Society, 13, 275-278.

(Manuscript received March 31, 2004; revision accepted for publication March 16, 2007.) 